\title{
Effects of short-term wear of silicone hydrogel contact lenses on refractive behaviour
}

\author{
WDH Gillan \\ Department of Optometry, University of Johannesburg, PO Box 524, Auckland Park, 2006 South Africa
}

Received 28 November 2011; revised version accepted 28 May 2012

<wgillan@uj.ac.za>

\begin{abstract}
Contact lens wear is known to induce change in both the cornea and refractive state. Often a shift towards increased myopia is noted. Historically investigations into the effects of contact lenses on refractive state have often been incomplete in terms of statistical analysis whereby nearest equivalent sphere is used or the spherical, cylindrical and axis components are analyzed in isolation. The aim of this study was to investigate the short-term effects of silicone hydrogel contact lenses on refractive behaviour. Seven volunteers agreed to wear a silicone hydrogel lens on one eye for a period of thirty min-
\end{abstract}

utes. Prior to lens wear, after ten minutes of lens wear and after thirty minutes of lens wear 50 autorefractor measurements were taken of refractive state from each subject. Data were analyzed using multivariate statistical methods. Scatter plots and other multivariate statistics are used to show how lens wear influences refractive behaviour. The results of this study show that silicone hydrogel contact lenses do influence refractive behaviour in both a spherical as well as an antistigmatic (astigmatism) fashion. (S Afr Optom 2012 71(2) 78-85)

Key Words: silicone hydrogel contact lenses, contact lenses, refractive behavior, autorefraction.

\section{Introduction}

It has been well documented over the years that contact lenses have an effect on, and can change, the state of refraction of an eye ${ }^{1-5}$. Rengstroff and Nilsson $^{2}$, using spherical equivalents to express their data, found that long-term extended wear of hydrogel lenses introduced clinically insignificant changes in refraction compared with refraction taken prior to extended wear of the lenses. Over seven days following removal of the extended wear lenses, however, there was a trend towards reducing levels of myopia as well as an increase in against-the-rule astigmatism. Phillips and Stone ${ }^{3}$ and Ruben and Guillon ${ }^{4}$ state that hydrogel lenses result in changes in the levels of myopia of wearers. Individuals wearing silicone lenses (as opposed to silicone hydrogels) showed little change in refraction while hydrogel lens wearers showed an in- crease in myopia during one year of lens wear ${ }^{5}$. Walline et $a l^{6}$ showed that over a three year period, soft contact lens wear resulted in more myopia progression than shown by rigid gas permeable lens wearers. A short-coming of early efforts to understand changes induced in refractive state when wearing hydrogel contact lenses was that incomplete methods of analyzing changes in refractive state were used (sphere and cylinder were analyzed separately or spherical equivalents were used). Gillan ${ }^{7}$ has shown evidence suggesting that silicone hydrogel contact lenses may result in increased levels of myopia over a six month wearing period. More recently studies investigating the effects of contact lenses on the higher-order aberrations of the eye have been published ${ }^{8-11}$. Clinically (and anecdotally) contact lens practitioners know that a contact lens takes time to settle once it has been placed on an eye. Just how long the lens takes to settle 
and the characteristics of the settling process have, as far as I know, not been investigated making use of the methods of analysis that are to be used in this study.

Making use of auto-refractors to determine refractive state is common practice among researchers ${ }^{12-17}$. The accuracy and validity of the autorefractor has also been shown ${ }^{12,15-16}$. The aim of this study was to investigate the effects that short-term wear of silicone hydrogel contact lenses might have on refractive behaviour.

\section{Method}

Seven volunteers (four female, three male), aged between 21 and 25, studying optometry at the University of Johannesburg were recruited for this study. Ethical clearance for the study was obtained. Informed consent was received from each subject after the purpose of the study was presented. Exclusion criteria included any ocular pathology, dry eye, contact lens wear and/or previous ocular surgery. Only one eye from each subject was fitted with a contact lens. The eye wearing the contact lens was chosen randomly. A Nidek ARK-700 autorefractor was used to take fifty measurements of refractive state prior to having a plano powered Bausch and Lomb Purevision 2 silicone hydrogel contact lens placed on the relevant eye. After ten minutes and at thirty minutes of lens wear a further fifty measurements of refractive state were obtained (while still wearing the contact lenses), resulting in a total of 150 measurements of refractive state for each subject. Subjects sat at leisure in the intervals between measurements. The autorefractor was refocused after each measurement and a print-out of the data was done after every ten measurements. The autorefractor was adjusted to measure refractive state at the corneal plane (vertex distance of zero), in $0.01 \mathrm{D}$ and $1^{\circ}$ increments. Fifty measurements of refractive state were taken of a test eye prior to any measurements being taken on subjects. This was done in an attempt to ensure accuracy of the autorefractor and to evaluate variation in the measurements taken of the test eye.

Refractive data were analyzed by converting measurements in sphere, cylinder and axis to component notation (stigmatic, ortho-anti-stigmatic and oblique anti-stigmatic components). Methods developed by Harris and Keating and software by Harris and Malan ${ }^{18-21}$, modified by Rubin, were used in the analysis of the data.

\section{Results}

Figure 1 shows a stereo-pair scatter plot of the fifty measurements taken of the test eye. By converging to a point in front of the page and fusing the two scatter plots, the reader will be able to perceive a three dimensional representation of the scatter plot (this applies to all scatter plots in this article). A tight cluster, seen as a black oval situated around the origin of the axes, contains the fifty measurements taken of the test eye. The origin of the axes represents the mean of the fifty measurements. Each axis is one dioptre in length. The size (and tightness) of the cluster suggests that little variation occurred in the 50 measurements taken of the test eye, especially when compared to the increased variation that took place in the measurements of refractive state of the subjects. Table 1 shows the descriptive statistics of the data collected from the test eye. The variance between the stigmatic, ortho-antistigmatic and oblique antistigmatic components is indicated by $\mathrm{S}_{11}, \mathrm{~S}_{22}$ and $\mathrm{S}_{33}$ while the stigmatic-ortho-antistigmatic, stigmatic-oblique antistigmatic and ortho-oblique antistigmatic covariances are shown by $\mathrm{S}_{12}, \mathrm{~S}_{13}$ and $\mathrm{S}_{23}$ respectively.
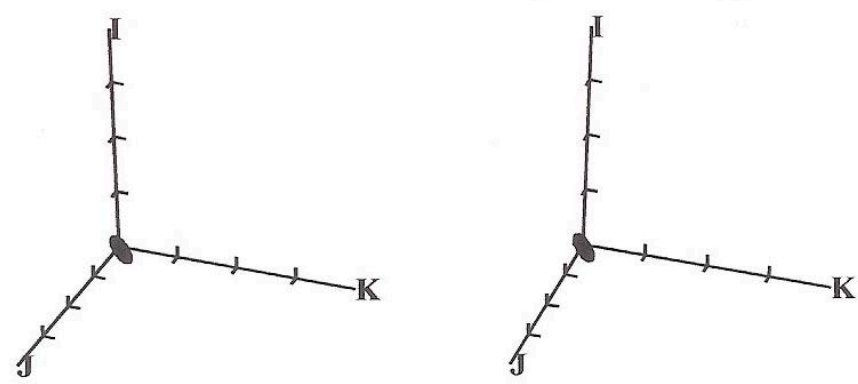

Figure 1: Stereo-pair scatter plot of fifty measurements of refractive state taken from a test eye. The origin is at the mean of the data and the axis length is one dioptre. By converging to a point in front of the page the reader will be able to fuse the two halves of the scatter plot and be able to appreciate a three dimensional percept of the figure (this applies to all scatter plots in this article). 
Table 1: Mean refractive state of the test eye given in conventional (sphere, cylinder and axis) and component notation (I, J and $\mathbf{K}$ ). Included are the variances and covariances.

\begin{tabular}{l|l|l|l|l|l|l|l} 
Conv. notation & Comp. notation & $\mathrm{S}_{11}$ & $\mathrm{~S}_{22}$ & $\mathrm{~S}_{33}$ & $\mathrm{~S}_{12}$ & $\mathrm{~S}_{13}$ & $\mathrm{~S}_{23}$ \\
\hline$-5.04-0.70 \times 95$ & $-5.04-0.03-0.01$ & 0.0004 & 0.000017 & 0.000019 & 0.00007 & -0.00017 & 0.00001
\end{tabular}

Figures 2 and 3 show stereo-pair scatter plots for the data collected from subjects 1 and 2. Data collected prior to lens wear are indicated by red points, data collected after ten minutes of lens wear by green points and data collected after thirty minutes of lens wear by blue points (this applies to all further scatter plots in this article). In both Figures 2 and 3 the data collected after ten minutes of lens wear (green points) appears different to the before and after thirty minutes of lens wear data. The difference is indicated by the altered orientation of the green ellipsoid (which is also positioned slightly higher than the remaining ellipsoids) in Figure 2 and the larger green ellipsoid (when compared with the red and blue ellipsoids) seen in Figure 3. The slightly higher position of the green ellipsoid in Figure 2 is an indication of a hyperopic increase in refractive state, a perception supported by the data in Table 2. The larger green ellipsoid in Figure 3 indicates increased variation in the measurements of refractive state collected from subject 2 after ten minutes of lens wear. The increased variation is also shown in Table 3 where the variance-covariances for the three sets of data collected from subject 2 are presented (the variance-covariance parameters for data collected after ten minutes of wear are greater than for the data collected before, and after thirty minutes, of wear). In both Figures 2 and 3 the red and blue sets of data are similar in terms of size and orientation.
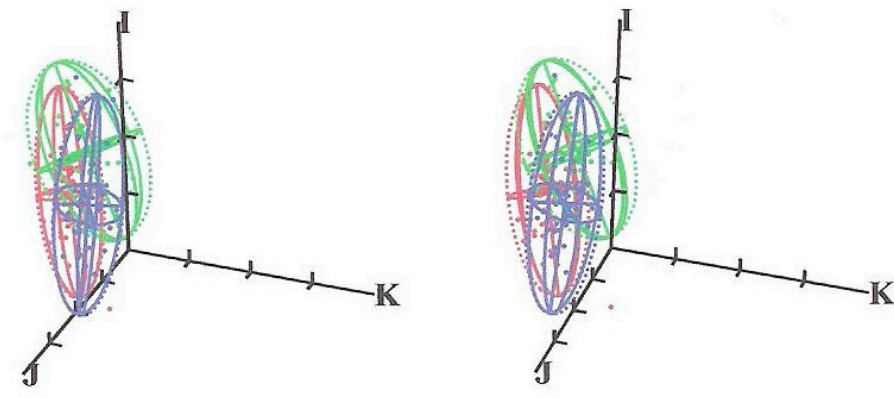

Figure 2: Stereo-pair scatter plot of fifty measurements of refractive state taken from subject 1 . The origin of the axes is at - 1I D. Red, green and blue data points represent measurements taken before, after ten minutes and after thirty minutes of lens wear respectively (this applies to all scatter plots in this article).
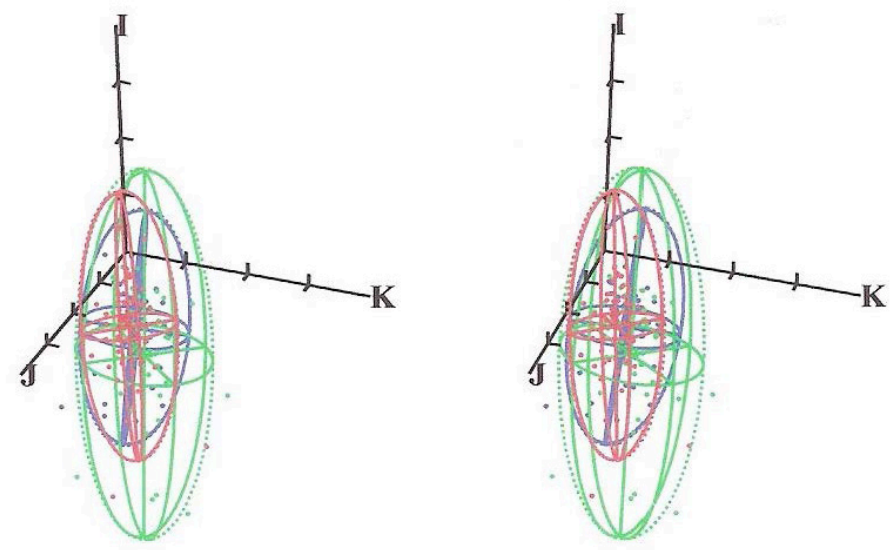

Figure 3: Stereo-pair scatter plot of fifty measurements of refractive state taken from subject 2 . The origin of the axes is at -1 I D.

Table 2: Mean refractive state for the seven subjects before contact lens wear, after ten minutes of contact lens wear and after thirty minutes of contact lens wear given in conventional notation (sphere, cylinder and axis) and in component notation. Lenses worn were plano Bausch and Lomb Purevision 2 silicone hydrogel lenses.

\begin{tabular}{l|l|l|l} 
Subject & Before wear & Ten minutes after & Thirty minutes after \\
\hline 1 & $-0.55-0.63 \times 123$ & $-0.45-0.31 \times 131$ & $-0.65-0.56 \times 118$ \\
& $-0.87-0.12-0.29$ & $-0.61-0.20-0.16$ & $-0.93-0.16-0.23$ \\
\hline 2 & $-0.82-0.64 \times 12$ & $-0.86-0.79 \times 16$ & $-0.70-0.80 \times 14$ \\
& -1.150 .290 .13 & -1.240 .320 .21 & -1.110 .360 .19 \\
\hline 3 & $-0.14-0.46 \times 11$ & $0.15-0.72 \times 5$ & $0.06-0.58 \times 12$ \\
& -0.370 .210 .09 & -0.200 .350 .09 & -0.230 .260 .11 \\
\hline 4 & $0.56-0.51 \times 7$ & $0.63-0.57 \times 5$ & $0.71-0.64 \times 2$ \\
& 0.310 .250 .06 & 0.340 .280 .05 & 0.390 .320 .02 \\
\hline 5 & $0.84-0.39 \times 10$ & $0.76-0.40 \times 2$ & $1.05-0.58 \times 172$ \\
& 0.640 .180 .07 & 0.550 .200 .01 & $0.760 .28-0.08$ \\
\hline 6 & $0.23-0.53 \times 89$ & $1.10-1.27 \times 80$ & $0.64-0.84 \times 83$ \\
& $-0.04-0.270 .02$ & $0.46-0.600 .22$ & $0.23-0.410 .10$ \\
\hline 7 & $0.74-0.10 \times 147$ & $0.97-0.36 \times 168$ & $-0.40-0.33 \times 179$ \\
& $0.690 .02-0.05$ & $0.790 .17-0.07$ & $-0.560 .16-0.01$
\end{tabular}


Table 3: Variance-covariance (units $D^{2}$ ) for the three sets of data collected from subject 2.

\begin{tabular}{l|l|l|l|l|l|l} 
Wear period & $\mathrm{S}_{11}$ & $\mathrm{~S}_{22}$ & $\mathrm{~S}_{33}$ & $\mathrm{~S}_{12}$ & $\mathrm{~S}_{13}$ & $\mathrm{~S}_{23}$ \\
\hline Before & 0.038 & 0.004 & 0.004 & -0.005 & -0.003 & -0.000 \\
After 10 & 0.071 & 0.012 & 0.011 & -0.009 & -0.001 & 0.003 \\
After 30 & 0.033 & 0.0036 & 0.006 & -0.002 & 0.003 & -0.000
\end{tabular}

Figures 4 and 5, showing scatter plots for data collected from subjects 3 and 4, reveal a different scenario. In both figures the green and blue ellipsoids are larger than the red ellipsoids, indicating that greater variation occurred in the measurements taken after ten and thirty minutes of lens wear. The size, shape and orientation of the green and blue ellipsoids are, respectively, similar to each other in the two figures. Table 2 shows the mean refractive state for each of the fifty measurements collected for subjects 3 and 4 . In both instances (Figures 4 and 5) the greatest variation in all sets of data is predominantly stigmatic (spherical) in nature (shown by the major axis of each ellipsoid being oriented along, or mostly along, the stigmatic axis). In both Figures 4 and 5 the "waist" of each green and blue ellipsoid is larger than the waist of the red ellipsoid (the minor diameter). The larger "waist" is indicative of a relative increase in the amount of anti-stigmatic variation that occurred in the measurements during and after lens wear (essentially an increase in the astigmatic component of the measurements was caused by the wear of this lens).

Figures 6,7 and 8 show the data collected from subjects 5, 6 and 7 respectively. Figure 6 shows an essentially spherical-appearing green ellipsoid suggesting that variation in measurements occurred in the stigmatic and antistigmatic plane equally. The red and blue ellipsoids have an elliptical shape with the predominant variation occurring along the stigmatic axis. The shapes of the ellipsoids suggest that the type of variation in refractive state before, after ten minutes and at thirty minutes was different. The other notable aspect of Figure 6 is that both the green and blue ellipsoids are larger than the red ellipsoid indicating greater variation in refractive measurements after ten and thirty minutes of lens wear. Figure 7 too indicates that greater variation occurred after ten and thirty minutes of lens wear. In Figure 7, however, the green and blue ellipsoids are larger than the red, suggesting much greater variation in refractive state af- ter ten and thirty minutes compared with that seen in Figure 6. Figure 8, showing data for subject 7 , has an axis length of $6 \mathrm{D}$ which was necessary for all three ellipsoids to be seen on one set of axes. Subject 7 manifested extreme variation in refractive state after thirty minutes of lens wear. The red and green ellipsoids are small and obscured by the blue ellipsoid. The blue ellipsoid (indicating measurements taken after thirty minutes of lens wear) is elongated predominantly along the stigmatic axis (suggesting primarily spherical variation). The length of the axis gives an indication of the magnitude of the variation in measurements. The descriptive statistics for subjects 5, 6 and 7 can be seen in Table 2 .
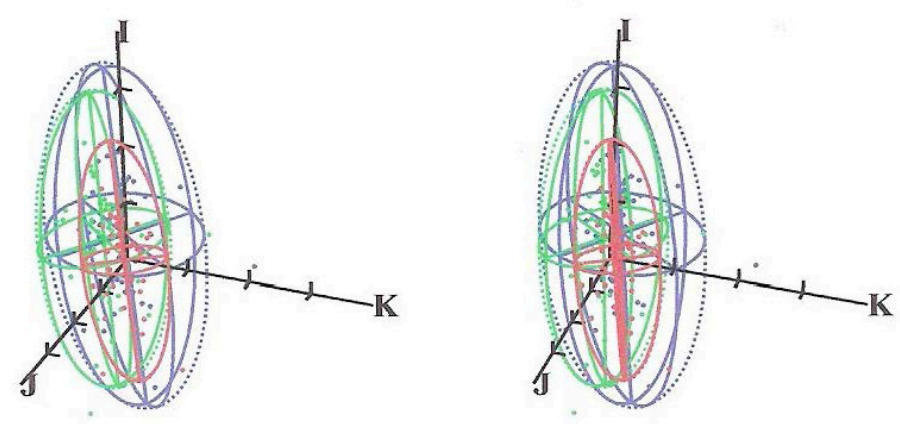

Figure 4: Stereo-pair scatter plot of fifty measurements of refractive state taken from subject 3 . The origin of the axes is at -0.5 I D.
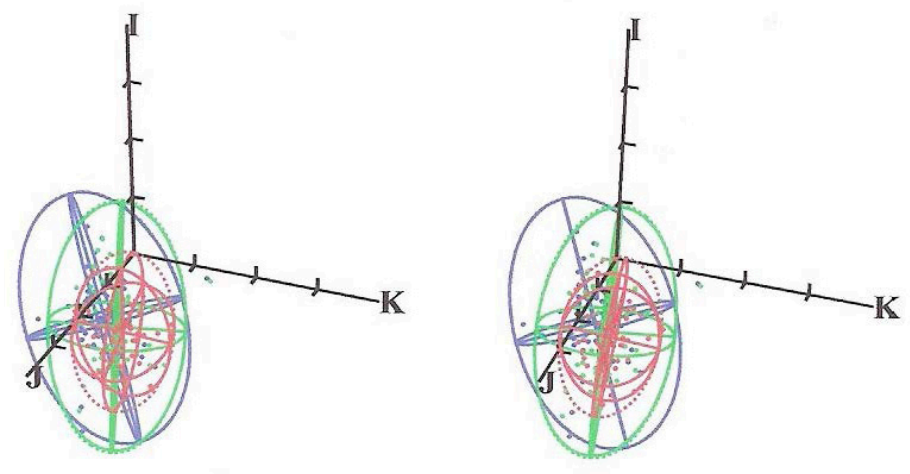

Figure 5: Stereo-pair scatter plot of fifty measurements of refractive state taken from subject 4 . The origin of the axes is at 0.5 I D. 

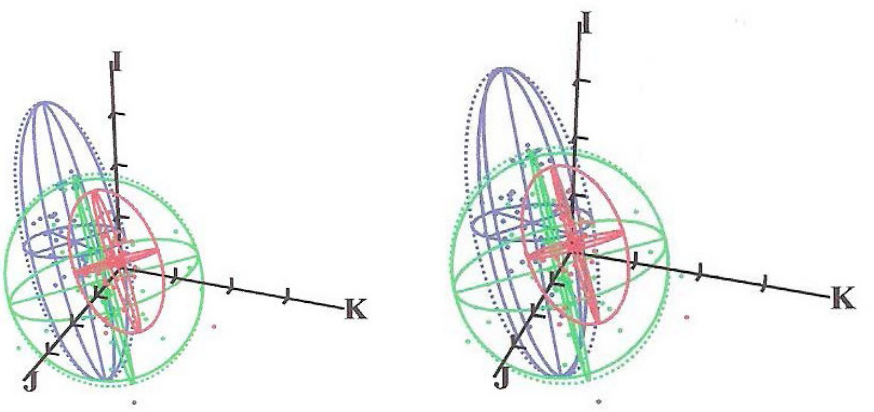

Figure 6: Stereo-pair scatter plot of fifty measurements of refractive state taken from subject 5 . The origin of the axes is at $0.5 \mathrm{I} \mathrm{D}$.
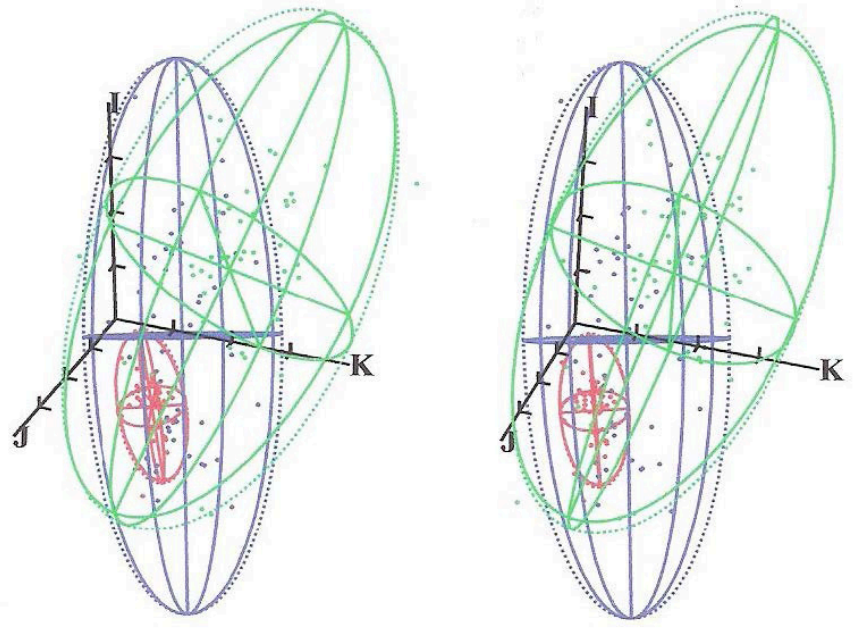

Figure 7: Stereo-pair scatter plot of fifty measurements of refractive state taken from subject 6 . The origin of the axes is at $0.5 \mathrm{I} \mathrm{D}$.
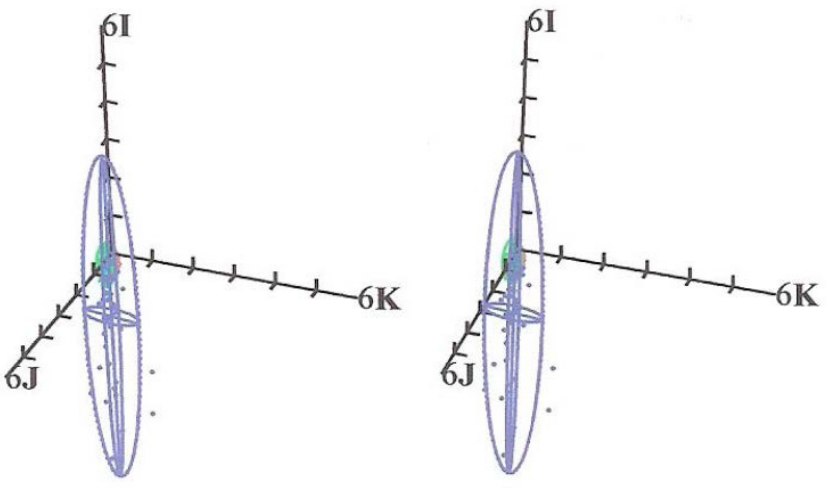

Figure 8: Stereo-pair scatter plot of fifty measurements of refractive state taken from subject 7 . The origin of the axes is at $1 \mathrm{I} \mathrm{D}$. Axis length is six dioptres, necessary to place all three ellipsoids on one set of axes. The red and green ellipsoids are partially obscured by the blue ellipsoid and are located near the origin of the axes.

A multivariate analysis of variance (MANOVA) was conducted on the data for each subject, testing the null hypothesis that there is no difference between the variancecovariances of the data collected before, after ten minutes and at thirty minutes of lens wear. The null hypothesis that there is no difference in variance-covariance between the before, after ten minutes and at thirty minutes of lens wear sets of measurements is rejected for all subjects.

A MANOVA was also conducted to test the null hypoth- esis that there is no difference between the means of the three sets of data collected from the seven subjects. The null hypothesis that there is no difference between the mean refractive states before, after ten minutes and after thirty minutes is rejected for all subjects.

Because of the large variation in measures of refractive state at thirty minutes of lens wear found in subjects 5, 6 and 7 it was decided to let these three subjects wear another brand of silicone hydrogel contact lens to see if similar changes occurred. The three subjects were fitted with Cooper vision Biofinity silicone hydrogel lenses.
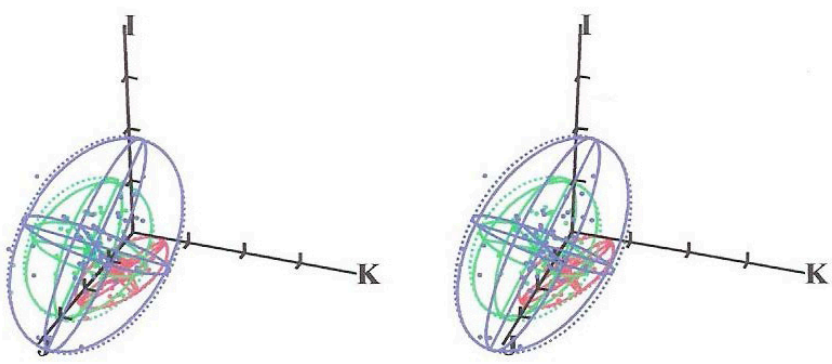

Figure 9: Stereo-pair scatter plot of fifty measurements of refractive stte taken from subject 5 wearing a Biofinity silicone hydrogel lens. The origin of the axes is at $0.5 \mathrm{I} \mathrm{D}$.
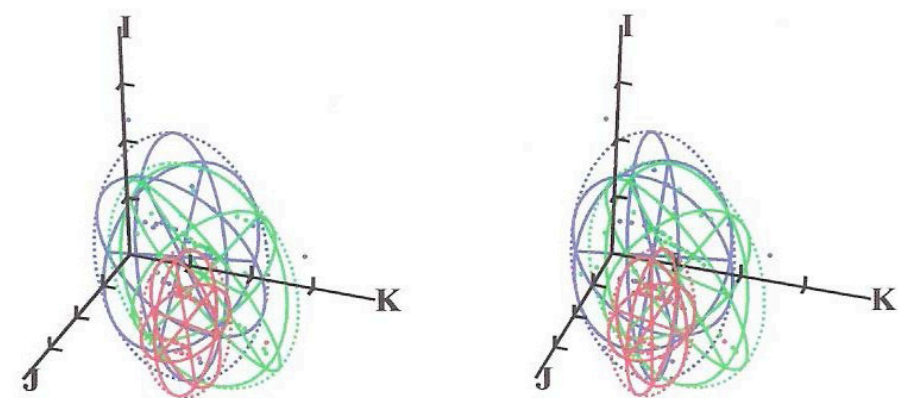

Figure 10: Stereo-pair scatter plot of fifty measurements of refractive state taken from subject 6 wearing a Biofinity silicone hydrogel lens. The origin of the axes is at $0.5 \mathrm{I} \mathrm{D}$.
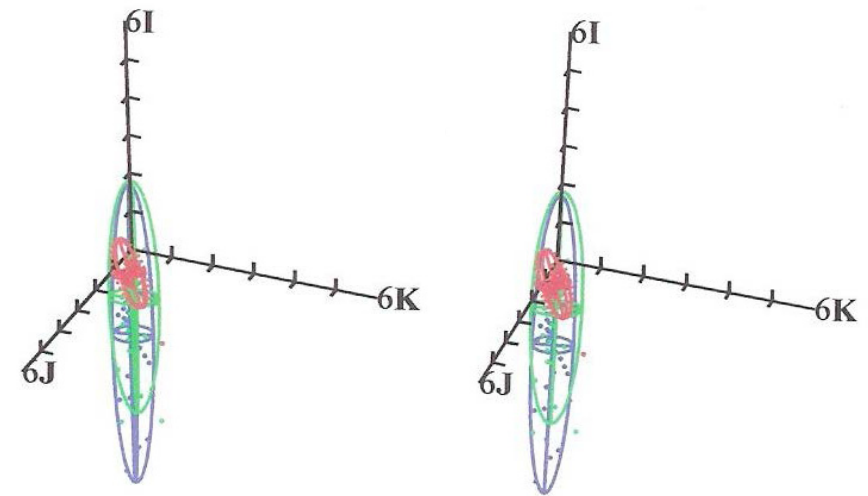

Figure 11: Stereo-pair scatter plot of fifty measurements of refractive state taken from subject 7 wearing a Biofinity silicone hydrogel lens. The origin of the axes is at $1 \mathbf{I} \mathrm{D}$. Axis length is six dioptres, necessary to place all thee ellipsoids on one set of axes. 
Table 4: Mean refractive state for three subjects (showing extreme changes wearing

\begin{tabular}{l|l|l|l} 
Subject & Before wear & Ten minutes after & Thirty minutes after \\
\hline 5 & $0.66-0.46 \times 6$ & $0.86-0.56 \times 173$ & $0.86-0.56 \times 175$ \\
& 0.440 .230 .05 & $0.560 .29-0.07$ & $0.580 .28-0.04$ \\
\hline 6 & $0.37-0.63 \times 83$ & $0.63-0.65 \times 73$ & $0.65-0.61 \times 83$ \\
& $0.05-0.300 .8$ & $0.30-0.270 .18$ & $0.35-0.300 .08$ \\
\hline 7 & $0.44-0.11 \times 157$ & $-0.21-0.06 \times 35$ & $-1.10-0.15 \times 2$ \\
& $0.380 .04-0.04$ & -0.240 .010 .03 & -1.170 .080 .01
\end{tabular}

Figures 9, 10 and 11 show scatter plots of data collected from subjects 5, 6 and 7, when wearing a Biofinity silicone hydrogel lens, respectively. Table 4 gives the mean refractive state, in conventional as well as component notation, for the three subjects before wear, after ten minutes of lens wear and at thirty minutes of lens wear. Figures 9, 10 and 11 show a similar pattern to that seen in Figures 6,7 and 8 where greater variation occurs in the fifty measurements taken after ten and thirty minutes of lens wear. A MANOVA was conducted testing whether the variance-covariances were different before, after ten minutes and after thirty minutes for the three subjects wearing the biofinity lenses. The null hypothesis that there is no difference in the variance-covariances is rejected for all three subjects.

MANOVA testing whether the means for the three subjects were different between the before, after ten minutes and after thirty minutes of lens wear allows the null hypothesis, that there is no difference between means, to be rejected for all three subjects.

\section{Discussion}

This study was conducted to investigate the effects of short-term wear of silicone hydrogel contact lenses on refractive behaviour. Seven subjects volunteered to wear Bausch and Lomb Purevision 2 silicone hydrogel lenses for a period of thirty minutes. Fifty measures of refractive state, using a Nidek ARK-700 autorefractor, were taken before lens wear, after ten minutes of lens wear and after thirty minutes of lens wear. A further fifty measurements of refractive state were taken, before any measures were taken from the seven subjects, of a test eye in order to evaluate the accuracy and repeatability of the autorefractor when measuring a static, inanimate test eye. The fifty measures of refractive state taken of the test eye show little variation, especially when compared with the vari- ation of the measurements obtained from the seven subjects. A stereo-pair scatter plot depicting the variation found in the test eye can be seen in Figure 1. The refractive state of the test eye is stated to be $-5.00 \mathrm{DS}$ by the manufacturer. The mean refractive state of the test eye as determined by fifty measures of refractive state using the autorefractor was found to be -5.04 -0.70 x 95 (see Table 1). The variance-covariance of the test eye data can also be found in Table 1. The data obtained from the test eye suggest that the autorefractor gives an accurate and repeatable indication of the refractive state as well as any variation that might be occurring in the test eye-autorefractor system. This contention is supported by other researchers ${ }^{12-17}$.

Figures 2 and 3 show scatter plots of the data collected from subjects 1 and 2 . The mean refractive state for these two subjects can be seen in Table 2. Both subjects 1 and 2 show increased, or altered, variation in the fifty measures of refractive state obtained after ten minutes of lens wear and this altered variation can be noticed in Figures 2 and 3. At thirty minutes of lens wear the scatter plots suggest that the character of the variation of the measurements is similar to the before measurements. This observation might be explained by the fact that the lenses take longer than ten minutes to settle on the eye. Figures 4 and 5 show the scatter plots for subjects 3 and 4 . In these two subjects there is increased variation in measurements of refractive state at ten minutes after, as well as thirty minutes after lens wear. The suggestion here is that subjects 3 and 4 take longer than thirty minutes for the lenses to settle on the eye. It is also possible that the greater variation found in these subjects at ten and thirty minutes of lens wear might remain and that the lenses might not settle adequately. Data collected from subjects 5, 6 and 7 are shown in Figures 6,7 and 8. In these instances the amount of variation occurring in the measures of refractive state taken at thirty minutes is greater than that seen in other subjects. Subjects 6 and 
7 reveal large changes in refractive variation in data collected at thirty minutes. The relative increases in variation can be seen in Figures 6, 7 and 8 when comparing the red, green and blue ellipsoids in the scatter plots. Figure 8 (subject 7) had to be plotted on axes that are $6 \mathrm{D}$ in length in order to fit all three ellipsoids on one set of axes. This suggests that extreme changes in variation of the measures of refractive state occurred at thirty minutes of lens wear (compare the red and green ellipsoids with the blue ellipsoid in Figure 8). A negative aspect of this study is that no attempt was made to measure the sight of the subjects (for example with a $\log M A R$ acuity chart) meaning that we have no idea of how this variation in measurements seen in subjects 5, 6 and 7 might affect their sight. MANOVA's were conducted investigating whether the variance-covariance and means of the data collected for the seven subjects were significantly different and both the means and the variance-covariances were found to be different for the seven subjects when wearing the Purevision 2 lenses. Subjects 5, 6 and 7, because of the large amount of change in refractive behaviour wearing the Purevision 2 lens, also wore Biofinity lenses for thirty minutes. Figures 9, 10 and 11 present the data collected from subjects wearing the Biofinity lens prior to, at 10 minutes and at thirty minutes of wear. Comparing Figures 6, 7, and 8 with Figures 9, 10 and 11 respectively one can see similar changes occurring in subjects 5, 6, and 7 wearing Biofinity lenses. However, the magnitude of the changes in subjects 5 and 6 was smaller when wearing the Biofinity lens. Subject 7 (Figures 8 and 11) showed increased change when wearing the Biofinity lens at the ten minute point compared to when wearing the Purevision 2 lens. The change in refractive behaviour at thirty minutes is similar. MANOVA was conducted on the data collected when wearing the Biofinity lens and the variance-covariances as well as the means were shown to be significantly different prior to, at ten minutes and at thirty minutes of lens wear. The influence of these changes (seen in subjects 5, 6 and 7 ) on the ability of the subjects to wear contact lenses successfully (in terms of visual acuity) would make an interesting study.

Negative aspects of this study include the following: subject numbers were small, no attempt was made to evaluate the fit of each lens (however, the chances that two silicone lenses, with limited base curve parameters, would both fit so poorly as to induce the changes shown here is highly unlikely), only silicone hydrogel lenses were used (how low Dk/L hydrogel lenses might influence refractive behaviour was not investigated), a control group who were measured before and after no lens wear was not used and no attempt was made to measure visual acuity with the lenses. Further research, taking cognisance of the limitations of this study, needs to be conducted to enhance our understanding of the effects of hydrogel contact lenses of refractive behaviour. The exact cause of, in some instances, large amounts of variation in measures of refractive state through silicone contact lenses is not clear at present. However, possibilities include: changes in the tear layer (on both surfaces of the lens), poor lens fit, blink characteristics and lens parameter changes.

In conclusion, this preliminary study has shown that silicone hydrogel contact lenses do have an effect on refractive behaviour in the short term with some subjects showing extreme changes induced by these lenses.

\section{Acknowledgements}

To Amy Kirton, Jonathan Mundell, Lisemarie de Coning, Sanet Strydom and Zelda Lotz for allowing me to use the data they collected. My thanks to Bausch and Lomb and Cooper vision for providing the contact lenses used. My appreciation to Professor A Rubin for providing software and advice.

\section{References}

1. Mandell RB. Contact lens practice: hard and flexible lenses $2^{\text {nd }}$ ed. Springfield: Charles C Thomas, 1976 364-367.

2. Rengstroff RH, Nilsson KT. Long-term effects of extended wear lenses: changes in refraction, corneal curvature and visual acuity. Am J Optom Physiol Opt 1985 62 66-68.

3. Phillips AJ, Stone J. Contact lenses $3^{\text {rd }}$ ed. London: Butterworths, 1989, pp 744-745.

4. Ruben M, Guillon M. Contact lens practice. London: Chapman and Hall, 1994, 1040-1042.

5. Hovding G. Variations of refractive error during the first year of contact lens wear. Acta Ophthal 198361 129-140.

6. Walline JJ, Jones LA, Mutti DO, Zadnik K. A randomized trial of the effects of rigid contact lenses on myopia progression. Arch Ophthalmol 2004122 1760-1766.

7. Gillan WDH. Refractive behaviour changes with six months daily wear of high and low oxygen permeability hydrogel contact lenses. S Afr Optom 200665 68-74. 
8. Hong X, Himebaugh N, Thibos LN. On-eye evaluation of optical performance of rigid and soft contact lenses. Optom Vis Sci 200178 872-880.

9. Jiang H, Wang D, Yang L, Xie P, He JC. A comparison of wavefront aberrations in eyes wearing different types of soft contact lenses. Optom Vis Sci 200683 769-774.

10. Efron S, Efron N, Morgan PB. Optical and visual performance of aspheric soft contact lenses. Optom Vis Sci 2008 85 201-210.

11. Keir N, Simpson T, Fonn D. Visual and optical performance of silicone hydrogel contact lenses for moderate myopia. $J$ Optom 20103 149-157.

12. McBrien NA, Millodot M. Clinical evaluation of the Canon autoref R-1. Am J Optom Physiol Opt 198562 786-792.

13. McKendrick AM, Brennan NA. Clinical evaluation of refractive techniques. J Am Optom Assoc 199566 758-765.

14. Goss DA, Grosvenor T. Reliability of refraction-a literature review. J Am Optom Assoc 199667 619-630.

15. Strang NC, Gray LS, Winn B, Pugh JR. Clinical evaluation of infrared autorefractors for use in contact lens over-refraction. Contact Lens Ant Eye 199720 137-142.

16. Mallen EAH, Wolffsohn JS, Gilmartin B, Tsujimura S. Clinical evaluation of the Shin-Nippon SRW-5000 autorefractor in adults. Ophthal Physiol Opt 200121 101-107.

17. Chat SWS, Edwards MH. Clinical evaluation of the ShinNippon SRW-5000 autorefractor in children. Ophthal Physiol Opt 200121 87-100.

18. Harris WF. Algebra of sphero-cylinders and refractive errors, and their means, variance and standard deviation. Am J Optom Physiol Opt 198865 794-802.

19. Harris WF. Mean of a sample of equivalent dioptric powers. Optom Vis Sci 199067 359-360.

20. Malan DJ. Dioptric power data analysis: computer implementation of graphical methods with clinical examples. $S$ Afr Optom $1993 \mathbf{5 2}$ 84-90.

21. Keating MP. An easier method to obtain sphere, cylinder and axis from an off-axis dioptric power matrix. Am J Optom Physiol Opt 198057 734-737. 